FIAN/TD-09/04

ITEP-TH-35/04

\title{
On distribution of number of trades in different time windows in the stock market.
}

\author{
I.M. Dremin ${ }^{(a)}$, A.V. Leonidov ${ }^{(a, b)}$ \\ (a) Theoretical Physics Department, P.N. Lebedev Physics Institute, Moscow, \\ Russia \\ (b) Institute of Theoretical and Experimental Physics, Moscow, Russia
}

\begin{abstract}
Properties of distributions of the number of trades in different intraday time intervals for five stocks traded in MICEX are studied. The dependence of the mean number of trades on the capital turnover is analyzed. Correlation analysis using factorial and $H_{q}$ moments demonstrates the multifractal nature of these distributions as well as some peculiar changes in the correlation pattern. Guided by the analogy with the analysis of particle multiplicity distributions in multiparticle production at high energies, an evolution equation relating changes in capital turnover and a number of trades is proposed. We argue that such equation can describe the observed features of the distribution of the number of trades in the stock market.
\end{abstract}

\section{Introduction}

Statistical analysis of the stock market properties undertaken from the physicist's point of view has drawn a lot of attention in the last decade. In fact, a large fraction of research in the new field of econophysics [1, 2, 3, 4] is devoted to questions related to finance. One of the central issues in description of the stock price dynamics is understanding the activity pattern characterized by the intensity of trading (number of trades in some given time interval). In particular, the long-range volatility correlations (one of the most important properties of the financial time series) are directly induced [5] by the long-range correlations in trading activity discussed in [6]. Very recently the multiscaling of the stock market activity was discussed, in the framework of a network approach to complex system dynamics, in [7. The analysis of the properties of the distribution of the number of trades has also allowed to prove that the trade-generating process is of distinctly non-markovian nature, 
so that the time at which the new trade occurs depends on the times of a lot of preceding trades [8, 9]. Therefore the study of higher-order correlations is of great importance.

In the present paper we perform a detailed analysis of the distributions of the number of trades in a set of chosen intraday time intervals. The technique used in our study is borrowed from the analysis of multiparticle production in high energy physics, see, e.g., the review papers [10, 11]. The analogy drawn is between the distribution of the number of trades in some chosen temporal interval (finance) and the distribution of the number of produced particles in some chosen rapidity (longitudinal momenta) interval, both referred to as multiplicity distributions. This technique allows to study correlations in the systems of many trades (particles). Both total and genuine (i.e., nonreducible to those of smaller groups of trades) correlations are considered for sets of any number of trades. The analysis made in the context of multiparticle production has revealed a number of remarkable properties of multiplicity distributions in different rapidity windows, in particular - their multifractal nature and a special behavior of genuine correlations governed by a spectacular dynamics of the particle production process. Therefore it is of obvious interest to repeat this analysis in the context of stock price dynamics, which is a main issue addressed in the present paper. Its results inspired us to formulate a dynamical model of trading activity in financial markets.

\section{Data processing}

The data we used in our analysis is the tick data for five stocks - EESR,MSNG, RTKM, SNGS and LKOH, traded at the Moscow International Currency Exchange (MICEX) in the year of 2003. For our study we have chosen five time intervals $\Delta T$ : 5 min., $15 \mathrm{~min} ., 45 \mathrm{~min}$. and $495 \mathrm{~min}$. (the trading day at MICEX).

Before turning to the analysis of the multiplicity distributions, let us discuss in some more details the above-mentioned analogy between the tradegenerating process in finance and multiparticle production in high energy physics.

In high energy physics one studies the particle production at some fixed collision energy. The higher the energy, the more particles are produced. In "financial" terms, one "invests" energy and gets particles from it. In financial context we suggest therefore an analogy between the energy and the total capital turnover (inflow for buy trades and outflow for the sell ones) in some chosen time interval. More precisely, we suggest an analogy between the collision energy $E_{\text {coll }}=\sqrt{s}$ (in the center of mass of colliding particles) and 
the capital turnover $\mathcal{J}$. The total capital turnover in larger temporal intervals is thus disseminated into those in smaller ones corresponding to particular distribution of capital in between the trades.

Let us illustrate the above-described analogy by considering the simplest characteristics relating the capital turnover and trade multiplicity distributions - a dependence of the mean number of trades in the time interval under consideration $\langle n\rangle_{\Delta T}$ on the capital turnover $\mathcal{J}_{\Delta T}$ (in multiparticle dynamics the corresponding quantity is thus an energy dependence of mean multiplicity which has been extensively studied, e.g., in [12, 13]). In Fig. 1] we show this dependence in the case of $\Delta T=45 \mathrm{~min}$.

We see that the mean multiplicity is a concave function of the capital turnover $\mathcal{J}$, i.e. $\left\langle N_{\Delta T}\right\rangle \propto \mathcal{J}^{\alpha}$ with $\alpha<1^{1}$. For other time intervals the dependence is similar.

Equivalently, for the logarithmic turnover $y \propto \ln \mathcal{J}$,

$$
\langle n\rangle \propto \exp (\alpha \cdot y)
$$

Let us now turn to the analysis of other properties of the trade multiplicity distributions. The corresponding distributions (normalized at the mean number of trades in each interval) for the five stocks considered are shown in Fig. 2 ,

From Fig. 2 we see, that normalizing the trade multiplicity distributions at the corresponding averages brings them to the scale-invariant (i.e. independent of the size of the time window) form. Let us also note that none of the standard distributions we have tried (Poisson, negative binomial, gamma, inverse gamma) give a satisfactory fit of the data showing that we are dealing with some complex trade-generating dynamics.

The properties of the trade multiplicity distributions are conveniently summarized by their suitably defined moments. Among the most important characteristics that are widely exploited, in particular, in analyzing the large multiplicity events in multiparticle production at high energies, are factorial moments (normalized and unnormalized), cumulants and their ratio, the socalled $H_{q}$ moments defined below.

The unnormalized factorial moments $\mathcal{F}_{q}$ with integer ranks $q \geq 1$ are defined by the following formula:

$$
\mathcal{F}_{q}=\sum_{n} P(n) n(n-1) \ldots(n-q+1)=\left.\frac{d^{q} G(z)}{d z^{q}}\right|_{z=1},
$$

\footnotetext{
${ }^{1}$ In multiparticle dynamics this ${ }^{*}$ would correspond to $\langle n\rangle \sim s^{\alpha / 2}-$ a dependence characteristic to, ${ }^{*}$ e.g., hydrodynamic models of particle production
} 
where $P(n)$ is the probability of having $n$ trades (the multiplicity distribution) for a given time interval.

The factorial moments (2) are, by definiton, always positive. It is easily seen that the average multiplicity is given by $\mathcal{F}_{1}$, the dispersion of the distribution is related to $\mathcal{F}_{2}$ etc. The higher is the rank of the moment $q$, the more important is the contribution coming from the tail of the distribution. They present therefore an ideal tool for focusing on the properties of the periods with intensive trading.

In equation (2) we have introduced a generating functional for the multiplicity distribution

$$
G(z)=\sum_{n=0}^{\infty} z^{n} P(n) .
$$

One often considers the normalized factorial moments

$$
F_{q} \equiv \frac{\mathcal{F}_{q}}{\langle n\rangle^{q}} \equiv \frac{\mathcal{F}_{q}}{\mathcal{F}_{1}^{q}}
$$

For Poisson distribution (independent trades) all normalized factorial moments are equal to 1 . Thus a dependence on the overall scale (mean multiplicity) is eliminated. Therefore the moments (4) provide a convenient basis for extracting information on the structure of correlations. Let us stress, that the factorial moments (both normalized and unnormalized) are characterizing the overall correlation pattern, where the contributions of genuine (irreducible) and reducible correlations are mixed. By genuine correlations we mean those in which all subsets of the analyzed system are interconnected. The reducible ones appear in those systems which can be split into two or more subsystems not connected to each other. The genuine irreducible correlations are described by the cumulants $\mathcal{K}$ :

$$
\mathcal{K}_{q}=\left.\frac{d^{q} \ln G(z)}{d z^{q}}\right|_{z=1}
$$

For example, if only two-point correlations are present, all cumulants with ranks $q>2$ are zero, etc. In complete absence of correlations (Poisson) all cumulants are zero.

In what follows we shall focus ourselves on the properties of the normalized factorial moments $F_{q}$. In Fig. 3] we show the dependence of $F_{q}$ on the rank $q$.

We see, that, apart from the small bending at small $q$, the logarithms of the normalized factorial moments are approximately proportional to the rank, $\ln F_{q} \propto q$, corresponding to an approximate exponential growth of the 
factorial moments. Let us note in passing, that already this excludes the negative binomial distribution, for which the growth of factorial moments with $q$ is faster than exponential.

Of special interest is, of course, a dependence of the moments on the temporal resolution $\Delta T$, revealing the scaling properties of the underlying trade multiplicity distributions. In particular, for generic fractal system one has $\ln F_{q}(\Delta T)=\alpha_{q} \ln \Delta T$. If $\alpha_{q} \propto q$, the dynamics is of monofractal nature. More complicated $q$-dependence of $\alpha_{q}$ indicates that we are dealing with a complex multifractal process. The dependence of the normalized factorial moments $F_{q}$ on $\Delta T$ is shown, for the moments with ranks in the interval $q=1 \cdots 10$, in Fig. 4

As mentioned above, the scaling pattern is described by the set of the slopes in Fig. 4. In Fig [5 we show the ratio of the corresponding average slopes to the rank $q$, i.e., $\alpha_{q} / q$.

The simple monofractal scaling would correspond to $\alpha_{q}(q) / q=$ const., while from Fig. [5 we clearly see that this is not the case thus pointing out to some complex multifractal pattern. In Fig. 4 we observe a characteristic strengthening of fluctuations (characterized by the normalized factorial moments) with increasing resolution $\Delta T$. The similar feature is well known in quantum chromodynamics $[14]^{2}$.

As mentioned above, the factorial moments describe the overall (both reducible and irreducible) correlation pattern. To characterize the irreducible content of the multiplicity distribution represented by cumulants, it is convenient to consider the moments $H_{q} \equiv \mathcal{K}_{q} / \mathcal{F}_{q}$ which represent a share of genuine correlations in their overall amount. They are more conveniently obtained from the factorial moments (44) by the recurrent relation

$$
H_{q}=1-\sum_{p=1}^{q-1} \frac{\Gamma(q)}{\Gamma(p+1) \Gamma(q-p)} \frac{F_{p} F_{q-p}}{F_{q}} H_{q-p} .
$$

The moments $H_{q}$, plotted in Fig. 6, show a very interesting behavior: they are changing sign at some $q$ ! This phenomenon was first predicted theoretically in multiparticle dynamics [20] where it was used as a tool allowing to uncover the details of the particle generation process. Afterwards it was confirmed by experiment [21, 22]. Let us stress, that when working with "usual" distributions like Poisson, negative binomial or gamma, the moments $H_{q}$ are non-negative. More exactly, they are zero for Poisson, positive for negative binomial and non-negative for gamma. To make it possible that

\footnotetext{
${ }^{2}$ Multifractality of the price-generating process was discussed, in a different context, in [15, 16, 17, 18, 19.
} 
$H_{q}$ become negative, one should consider, at least, a mixture of these standard distributions, see [23. Physically, positive cumulants (and thus positive $H_{q}$ ) correspond to the effective repulsion between the points, and negative ones - to their attraction (clustering of trades). Changing sign means passing through the "Poissonian" point, where the corresponding irreducible contribution is absent. Below we shall consider a phenomenological dynamical model of the capital turnover that allows to describe this phenomenon in the financial context.

\section{Mathematical model and its predictions}

The above analysis of the stock market data allows to formulate a mathematical model describing the trade multiplicity distributions inspired by ideas originating from high energy physics. As already mentioned, the method of correlation analysis discussed below was first suggested in [20] and is widely used in the studies of multiparticle dynamics in high energy physics (see, e.g., a review article [11]). In multiparticle dynamics one considers multiplicity distributions in some region of phase space at given collision energy. Particle creation is described within well-established and well-tested theory of strong interactions - quantum chromodynamics. There exist sufficiently rigorous equations and well-defined predictions for the characteristics of interest. Among them are those for the energy dependence of mean multiplicity, multifractal properties of the particle production process and specific behavior of $H_{q}$ moments. We have observed the similar regularities in the trade distributions analyzed above.

As has been already mentioned, in particular, the change in the sign of $H_{q}$-moments with growing $q$ in multiparticle dynamics was theoretically predicted [20] and afterwards confirmed experimentally [21, 22]. It has been extensively studied theoretically both for the electron-positron collisions [24, 25. 26] and for hadron-hadron ones [23]. Let us note, that for standard probability distributions such as the negative binomial one this effect does not take place. This points out to a quite peculiar underlying dynamics both in multiparticle production and trading in the stock market.

Let us try to write down phenomenological equations for the generating functional $G(z, y)$ for the probability distribution of the trade multiplicity at given logarithmic turnover $y=\ln \mathcal{J}_{\Delta T} / \mathcal{J}_{0}\left(\mathcal{J}_{0}=\right.$ const):

$$
G(z, y)=\sum_{n=0}^{\infty} z^{n} P(n, y)
$$

In Eq. (7) the dependence on $y$ is shown explicitely (while it was not 
indicated in (3) $)$.

Following the analogy with quantum chromodynamics, we write

$$
\frac{d G}{d y}=\gamma_{0}^{2} \int_{0}^{1} d x K(x)[G(y+\ln x) G(y+\ln (1-x))-G(y)] .
$$

Equation (8) can be interpreted as follows. What we are trying to establish is a relation between the distribution of trades in the intervals with differing capital turnover. The relation can be described as an evolution from smaller to larger capital turnovers. It proceeds through adding "extra" turnover, of fractional size $x$, to the current one with fraction $1-x$ forming the total turnover given by $y$ - thus the quadratic structure in the RHS of (8) , describing the inflow to the total turnover. The linear term with negative sign corresponds to the outflow from the total turnover and therefore it depends on $y$ only. The weight of changing some fraction of the existing capital is specified by the kernel $K(x)^{3}$. In equation (8) the constant $\gamma_{0}$ describes the strength of the overall evolution rate in a financial market and the integration is over the splitting fraction $x$. The conservation of turnover is due to the arguments $\ln x$ and $\ln (1-x)$ in the right hand side.

Let us assume that the kernel $K(x)$ has the following functional form:

$$
K(x)=\frac{1}{x}-c-d x,
$$

where $c$ and $d$ are constants. The $1 / x$ term corresponds to the leading role of the trades having parametrically small contribution to the capital turnover ${ }^{4}$. It is tempting to assume that this effect can be related to the $1 / f$ behavior found in the analysis of spectral densities of daily trades [6]. The constant and linear terms describe the impact of large trades. In principle, next terms of the Laurent expansion of the kernel can contribute as it happens in quantum chromodynamics. However, in characteristics to be considered, it turns out that by keeping the $c$ - and $d$ - terms in the kernel one can conveniently parametrize all important effects. The equation (8) with the kernel (91) can be solved explicitely (see, e.g., [13]). For the mean multiplicity the exponential dependence on $y$ follows directly if the coupling strength $\gamma_{0}$ does not depend on the capital turnover. Some non-linearity in the exponent can be introduced by imposing such a dependence as it happens in quantum chromodynamics (see, e.g., [12]). The equation (8) also reveals the multifractal properties of the distributions [14] noticed by us in the above analysis.

\footnotetext{
${ }^{3}$ Note that for technical reasons it is convenient to work with the splitting variable $x$ denoting the relative yield of the incoming capital in the newly formed (i.e., total) one.

${ }^{4}$ In particle physics this corresponds to an emission of particles having small energies the so-called "infrared" particles.
} 
To proceed with $H_{q}$-moments, let us rewrite equation (7) as an expansion in the normalized factorial moments $F_{q}$ :

$$
G(z, y)=\sum_{q=0}^{\infty} z^{q}\langle n\rangle^{q} F_{q} .
$$

Assuming that the dependence on the capital turnover $y$ is coming only through the average multiplicity $\langle n\rangle$

$$
\langle n\rangle \propto \exp (\gamma y)
$$

we obtain the following equation for the factorial moments ${ }^{5}$ :

$$
\gamma q F_{q}=\gamma_{0}^{2} \int_{0}^{1} d x K(x)\left[\sum_{l=0}^{q} x^{\gamma l}(1-x)^{\gamma(q-l)} F_{l} F_{q-l}-F_{q}\right] .
$$

Equation (12) is solved iteratively starting from $q=1$. At $q=1$ one gets the following equation for the rate $\gamma$ of the growth of mean multiplicity with increasing capital turnover $\left(F_{0}=F_{1}=1\right)$ :

$$
\gamma=\gamma_{0}^{2} \int_{0}^{1} d x K(x)\left[x^{\gamma}+(1-x)^{\gamma}-1\right] .
$$

From (13) one can find $\gamma$ as a function of $\gamma_{0}$ and $c$. With good accuracy the corresponding equation can be reduced to an algebraic one:

$$
\gamma^{2}=\gamma_{0}^{2}\left[1+\left(C-c-\frac{d}{2}\right) \gamma-\left(\frac{\pi^{2}}{6}-2 c-\frac{d}{2}\right) \gamma^{2}\right],
$$

where $C \approx 0.577$ is Euler constant. At $\gamma \ll 1$ one has $\gamma \approx \gamma_{0}$. More accurately,

$$
\gamma \approx \gamma_{0}\left[1+\frac{1}{2}\left(C-c-\frac{1}{2} d\right) \gamma_{0}\right]
$$

The magnitude of $\gamma$ determines the growth of the average trade multiplicity $\langle n\rangle \propto \exp (\gamma y)$ with $y$. In the weak coupling regime $\gamma$ is practically equal to $\gamma_{0}$.

To understand the behavior of the moments $H_{q}$, let us first consider the case of $c=d=0$. Then the contribution to the RHS of the evolution equation (12) is dominated by small $x$, and $G(y+\ln (1-x))$ can simply be replaced by $G(y)$, so that

$$
(\ln G)^{\prime}=\gamma_{0}^{2} \int_{0}^{1} \frac{d x}{x}[G(y+\ln x)-1]=\gamma_{0}^{2} \int_{-\infty}^{y} d z[G(z)-1] .
$$

\footnotetext{
${ }^{5}$ Technically the condition of the approximate $F$-scaling $F_{q}^{\prime} \ll \gamma q F_{q}$ should hold.
} 
Differentiating both sides of (15) over $y$, we have

$$
(\ln G)^{\prime \prime}=\gamma_{0}^{2}[G-1] .
$$

Using Eqs (2), (15), one gets at $\gamma \approx \gamma_{0}$

$$
H_{q}=\frac{\gamma_{0}^{2}}{\gamma^{2} q^{2}} \approx \frac{1}{q^{2}}
$$

From Eq. (17) we conclude that the $H_{q}$-moments are always positive if there are small contributions to the capital turnover only.

Taking into account the remaining terms in the kernel $K(x)$, we get

$$
H_{q}=\frac{\gamma_{0}^{2}}{\gamma^{2} q^{2}}\left(1+\left(C-c-\frac{1}{2} d\right) q\right) .
$$

Now, $H_{q}$ change sign at some value of $q$. We see, that the zero of the $H_{q}$ moments is located at $q=\left(c+\frac{1}{2} d-C\right)^{-1}$. At larger $q$ the moments $H_{q}$ are negative and reach their minimum at some $q_{\min }$. The larger is the contribution of "strong" dealers (large $c$ and (or) $d$ ) the lower is the intercept of $H_{q}$ with the $q$-axis. In our analysis of stock data we have just met with such a behavior. Let us also note, that from Fig. (6) we see, that the data with lower two-point $\left(H_{2}\right)$ correlation have a smaller intercept of $H_{q}$. This implies that "strong" dealers are more influential in markets (or time intervals) characterized by weak interaction with other market participants. Various stocks differ in their relative roles - as seen from comparison of various plots in Fig. (6). It would certainly be of interest to compare these intercepts for different markets. This is an important signature of long-range correlations involved.

To make the above analysis more exact, a numerical recursive solution of (12) is required. For example, at $q=2$ we get the solution in the form

$$
F_{2}=\frac{\int_{0}^{1} d x K(x) x^{\gamma}(1-x)^{\gamma}}{2 \int_{0}^{1} d x K(x)\left[x^{\gamma}+(1-x)^{\gamma}-1\right]-\int_{0}^{1} d x K(x)\left[x^{2 \gamma}+(1-x)^{2 \gamma}-1\right]}
$$

In the above qualitative argumentation we have used, following the analogy with QCD, the small - $\gamma$ approximation. To get a realistic description of financial data in question one has to perform a deeper analysis of the formulae describing, e.g., the $H_{q}$ moments in the suggested formalism. At present, we limit ourselves by these qualitative statements. Quantitative estimate of theoretical parameters from the data and its implications will follow. 


\section{Conclusions}

We have analyzed the tick data for five stocks traded at MICEX and found some common qualitative features for all of them. The most important ones are:

1. The normalized trade multiplicity distributions are, to a good accuracy, scale-invariant with respect to the size of the time window considered.

2. The average multiplicity of trades is a concave function of the total capital turnover.

3. The multiplicity distributions demonstrate a multifractal behavior.

4. The cumulant (and therefore $H_{q}$ ) moments change their sign at some rank.

These features are strongly reminiscent of the properties of particle multiplicity distributions studied in high energy physics where the well-developed theory of quantum chromodynamics describes (and moreover predicts) them quite successfully. Using this analogy, we have proposed the phenomenological model of trades multiplicity distribution with the equation similar to that arising in quantum chromodynamics. This equation provides a qualitative description of the above-mentioned properties. Its interpretation in terms of inflow and outflow of the capital turnover, providing some insight into the dynamics of financial markets, is considered.

The work was supported by RFBR grants 04-02-16880, 04-02-16445-a, 02-02-16779 and the Scientific Schools Support Grant 1976.2003.02.

\section{References}

[1] R.N. Mantegna, H.E. Stanley, An Introduction to Econophysics, Cambridge, 2000.

[2] J.-P. Bouchaud, M. Potters, Theory of Financial Risk and Derivative Pricing, Cambridge, 2003.

[3] M.M. Dracorogna, R. Gencay, U. Muller, R.B. Olsen and O.V. Pictet, An Introduction to High Frequency Finance, Academic Press, 2001.

[4] J. Voit, The Statistical Mechanics of Financial Markets, Springer, 2003 
[5] V. Plerou, P. Gopikrishnan, L.A. Nunes Amaral, X. Gabaix, H.E. Stanley, "Economic Fluctuations and Diffusion", Phys. Rev. E62 (2000), R3023; e-print arXiv:cond-mat/9912051.

[6] G. Bonanno, F. Lillo and R. Mantegna, "Dynamics of the Number of Trades of Financial Sequrities", Physica A280 (2000), 136; e-print arXiv:cond-mat/9912006.

[7] Z. Eisler, J. Kertesz, S.-H. Yook, A.-L. Barbasi, "Multiscaling and non-universality in fluctuations of driven complex systems", e-print arXiv:cond-mat/0408409

[8] A. Leonidov, "Long Memory in Stock Trading", Int. Journ. of Theor. and Appl. Finance $\mathbf{7}$ (2004), 1; e-print arXiv:cond-mat/0303222.

[9] A. Leonidov, "On Non-Markovian Nature of Stock Trading", e-print arXiv:cond-mat/0403469.

[10] E.A. De Wolf, I.M. Dremin, W. Kittel, "Scaling Laws for Density Correlations and Fluctuations", Phys. Repts 270 (1996), 1; e-print arXiv: hep-ph/9508325.

[11] I.M. Dremin, J.W. Gary, "Hadron Multiplicities", Phys. Repts 349 (2001), 331; e-print arXiv:hep-ph/0004215

[12] I.M. Dremin, V.A. Nechitailo, "Average multiplicities in gluon and quark jets in higher-order perturbative QCD", Mod. Phys. Lett. A9 (1994), 1471.

[13] I.M. Dremin, R.C. Hwa, "Average multiplicities in gluon and quark jets as exact solution of QCD equations", Phys. Lett. B324 (1994), 447.

[14] Yu.L. Dokshitzer, I.M. Dremin, "Multiplicity fluctuations within small phase space bins in QCD", Nucl. Phys. B402 (1993), 139.

[15] A. Arneodo, J-F. Muzy, D. Sornette, "Causal cascade in the stock market from the 'infrared' to the 'ultraviolet'", Eur. Phys. J. B2 (1998), 277; e-print arXiv:cond-mat/9708012.

[16] J.-F. Muzy, J. Delour, E. Bacry, "Modelling fluctuations of financial time series: from cascade process to stochastic volatility model", Eur. Phys. J. B17 (2000), 537; e-print arXiv:cond-mat/0005400.

[17] E. Bacry, J. Delour, J.-F. Muzy, "Modelling financial time series using multifractal random walk", Physica A299 (2001), 294. 
[18] J.-P. Bouchaud, M. Potters, M. Meyer, "Apparent multifractality in financial time series", Eur. Phys. J. B13 (1999), 595; e-print arXiv:cond-mat/9906347.

[19] K. Matia, Y. Ashkenazy, H.E. Stanley, "Multifractal Properties of Price Fluctations of Stocks and Commodities", Europhys. Lett. 61 (2003), 422; e-print arXiv:cond-mat/0308012.

[20] I.M. Dremin, "Cumulant and factorial moments in perturbative gluodynamics", Phys. Lett. B313 (1993), 209.

[21] I.M. Dremin, V. Arena, G. Boca et al, "Cumulant to factorial moment ratio and multiplicity data", Phys. Lett. B336 (1994), 119; e-print arXiv: hep-ex/9405007.

[22] SLD Collaboration, "Factorial and cumulant moments in $e^{+} e^{-}$to hadrons at $Z^{0}$ resonance", Phys. Lett. B371 (1996), 149; e-print arXiv: hep-ex/9601010.

[23] I.M. Dremin, V.A. Nechitailo, "Independent pair parton interactions model of hadron interactions", Phys. Rev. D70 (2004), 034005; e-print arXiv:hep-ph/0402286

[24] I.M. Dremin, V.A. Nechitailo, "Moments of multiplicity distributions in higher-order perturbative QCD", JETP Lett. 58 (1993), 881.

[25] I.M. Dremin, R.C. Hwa, "Quark and gluon jets in QCD: factorial and cumulant moments", Phys. Rev. D49 (1994), 5805.

[26] M.A. Buican, C. Förster, W. Ochs, "QCD explanation of oscillating hadron and jet multiplicity moments", Eur. Phys. J. C31 (2003), 57; e-print arXiv:hep-ph/0307234. 

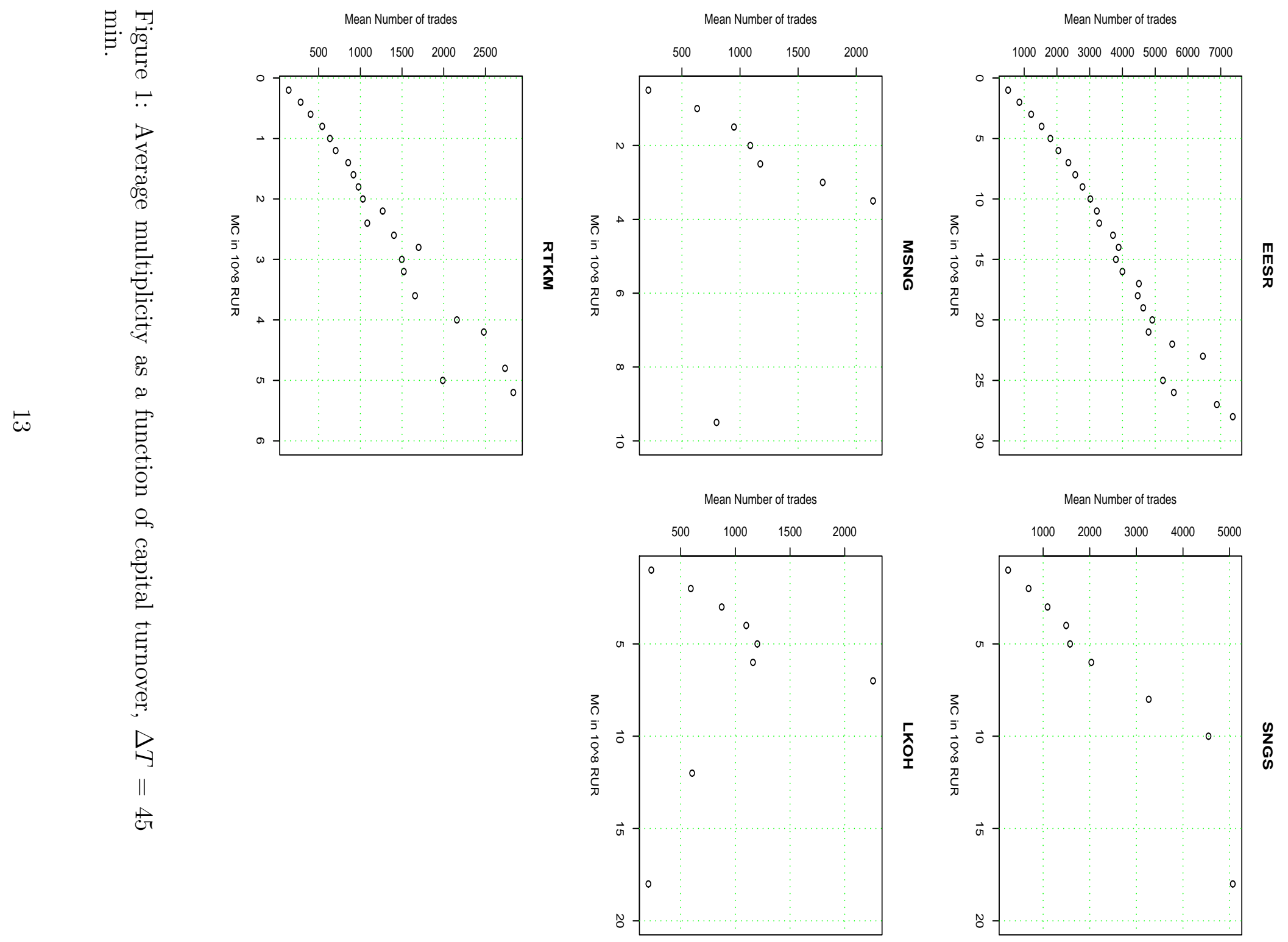

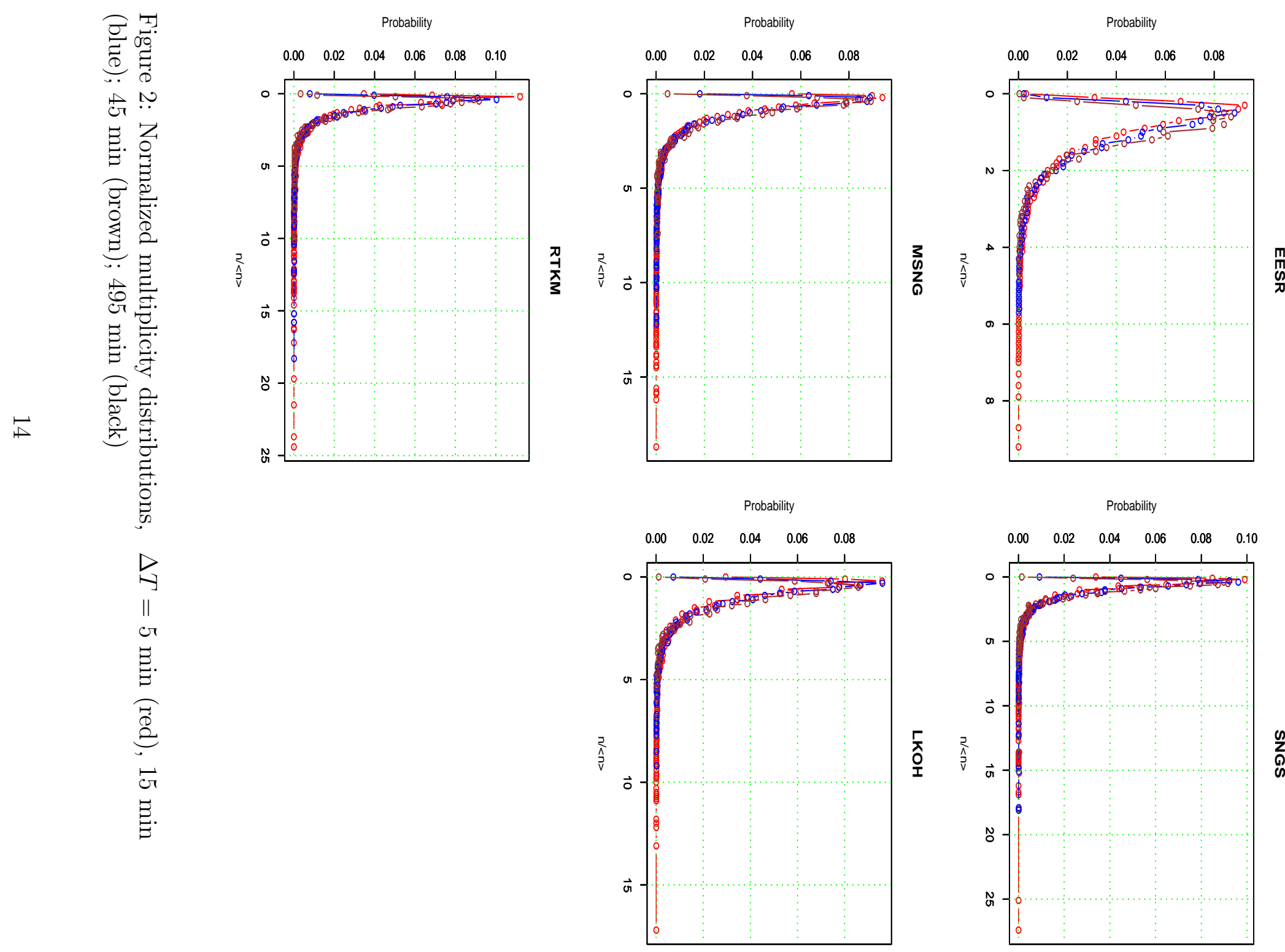

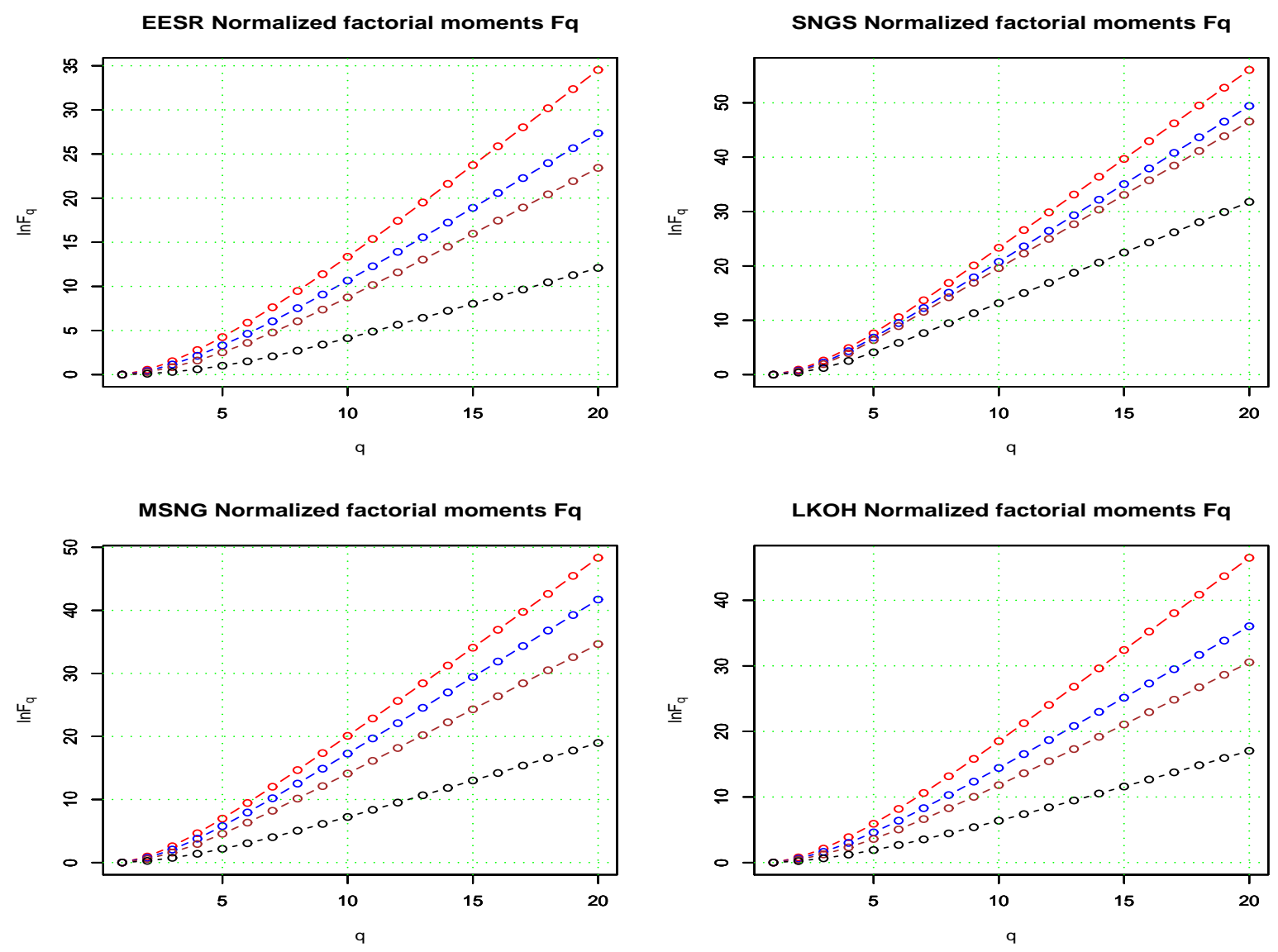

RTKM Normalized factorial moments Fq

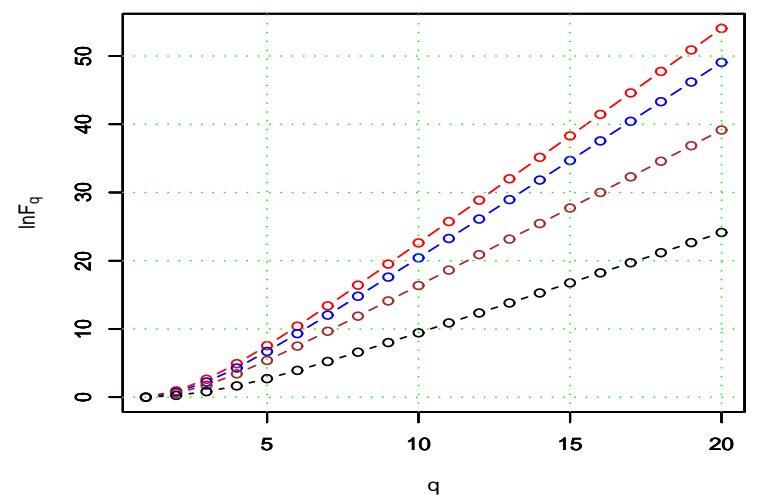

Figure 3: Normalized factorial moments $F_{q} ; \Delta T=5$ min (red), 15 min (blue); 45 min (brown); 495 min (black). 

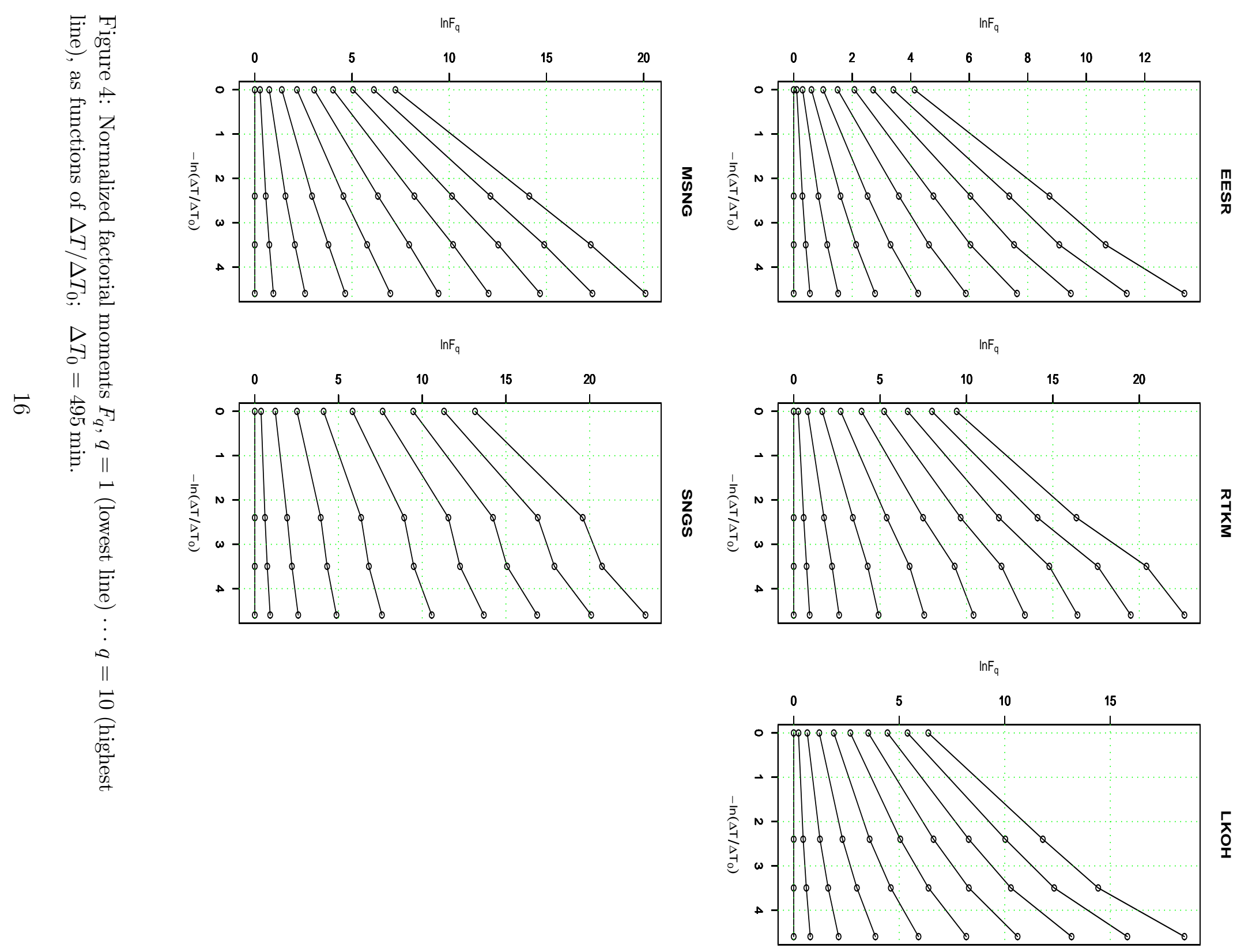


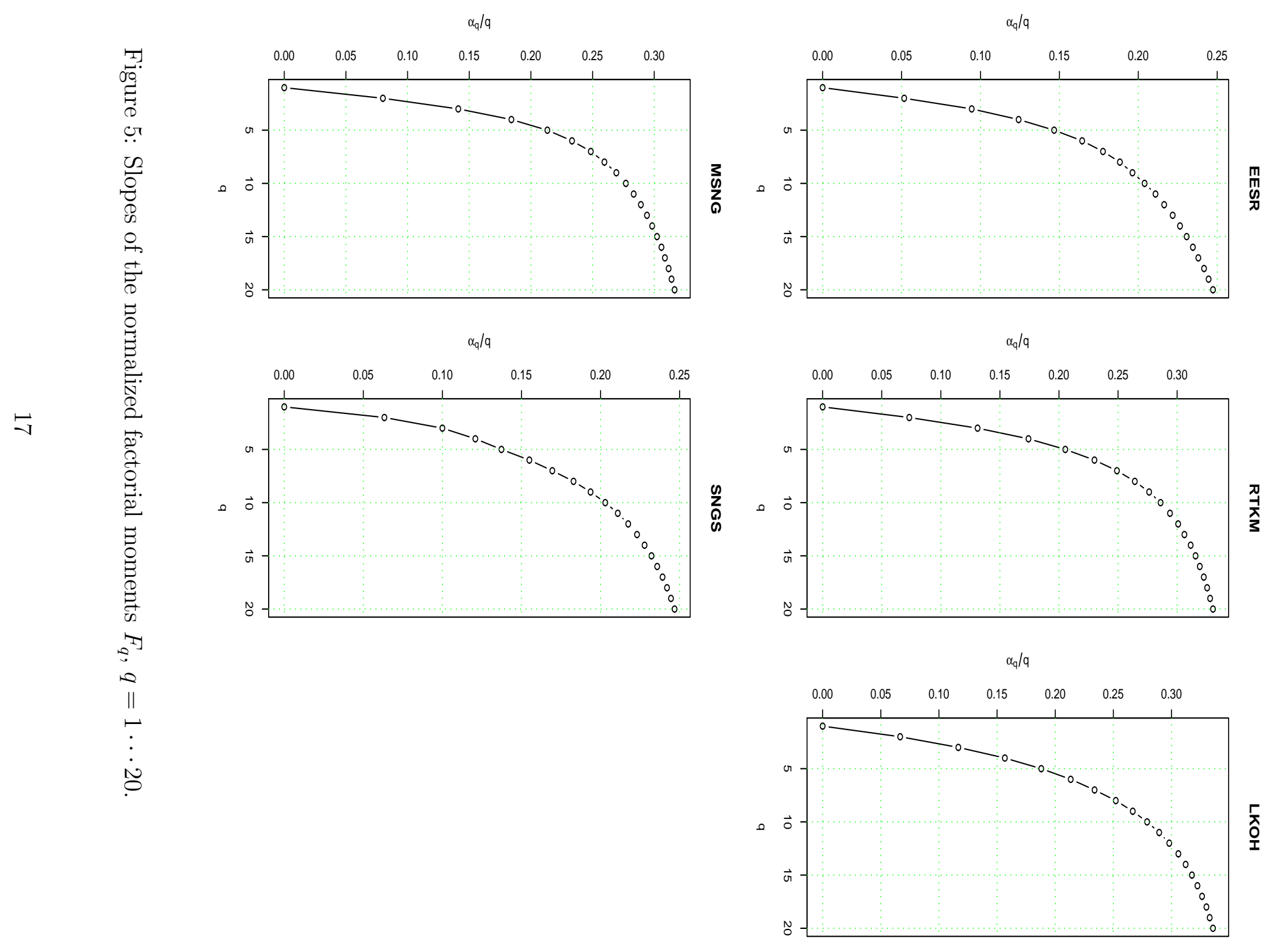



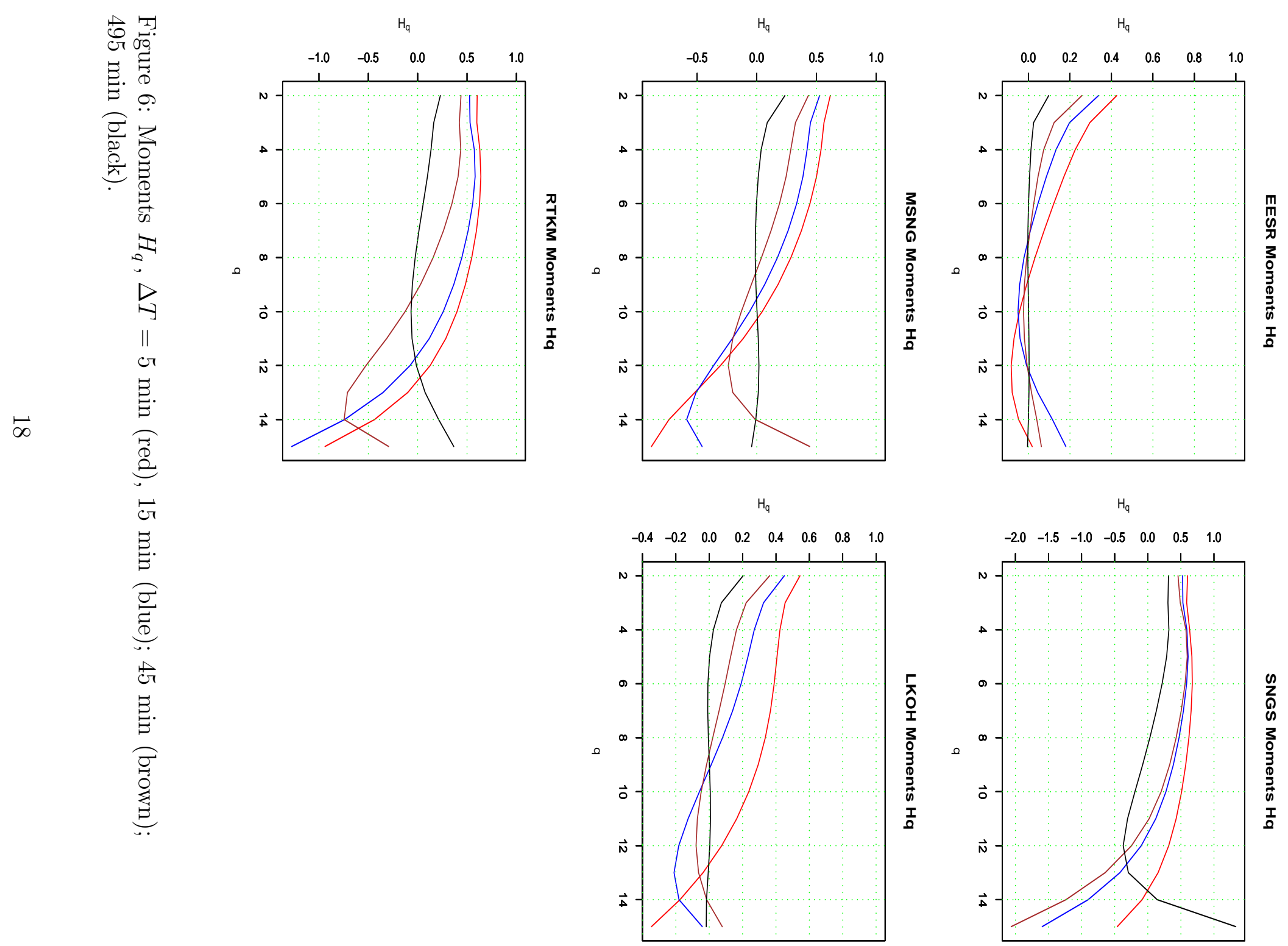